\title{
Large-scale simulations of antihelium production in cosmic-ray interactions
}

\section{Anirvan Shukla, ${ }^{a, *}$ Amaresh Datta, ${ }^{b}$ Philip von Doetinchem, ${ }^{a}$ Diego-Mauricio Gomez-Coral $^{a}$ and Carina Kanitz ${ }^{c}$}

${ }^{a}$ Department of Physics and Astronomy, University of Hawai'i at Mānoa, 2505 Correa Road, Honolulu, USA

${ }^{b}$ Joint Institute for Nuclear Research, 6 Joliot-Curie Street, Dubna, Russia

${ }^{c}$ Erlangen Center for Astroparticle Physics, Friedrich-Alexander-Universität Erlangen-Nürnberg, Erwin-Rommel-Str. 1, 91058 Erlangen, Germany

E-mail: anirvan@hawaii.edu

The possibility of antihelium production in interaction of cosmic rays with the interstellar gas is studied using large-scale Monte Carlo simulations. For this purpose, an energy-dependent coalescence mechanism developed previously is extended to estimate the production of light antinuclei $\left({ }^{3} \overline{\mathrm{He}}\right.$ and $\left.{ }^{4} \overline{\mathrm{He}}\right)$. The uncertainty in the coalescence parameter and its effect on the expected antiparticle flux is also investigated. The simulated background antihelium fluxes are found to be lower than the fluxes predicted by simplified models using numerical scaling techniques.

$37^{\text {th }}$ International Cosmic Ray Conference (ICRC 2021)

July 12 th - 23rd, 2021

Online - Berlin, Germany

\footnotetext{
${ }^{*}$ Presenter
} 


\section{Introduction}

Cosmic-ray (CR) antinuclei are a potential breakthrough approach for dark matter searches, because dark matter induced CR antinuclei fluxes predicted by many different models exceed the predicted astrophysical background in the energy range of $\mathrm{GeV}$ or sub-GeV by multiple orders of magnitude [1].

The space-based AMS-02 experiment [5] recently reported several antihelium candidates [6]. Naively, this implies that antideuterons should be observable in large quantities as well. However, thus far, no antideuteron candidates have been reported. These unexpected antihelium observations have therefore spurred an interest in studying the secondary production and propagation of antihelium in our Galaxy. Most studies have relied on simplified numerical scaling of antiproton production cross sections to predict the production cross sections of heavier antinuclei in typical CR-ISM interactions $[3,6,7]$.

A different approach is to use an event-by-event implementation of the coalescence model [812]. In Ref. [13], (anti)deuteron production measurements for proton-proton collisions at different energies were fitted with coalescence simulations to determine the best-fit energy-dependent parametrization for the coalescence momentum $p_{0}$. In this study, that $p_{0}$ parametrization was used to further develop a multiparticle coalescence mechanism. This approach benefits from the continuous improvement of Monte Carlo (MC) particle interaction simulators; the development of an event-by-event afterburner; and, finally, the availability of high-throughput computational facilities. Utilizing massive computation power of 6,000 years of CPU time, more than 30 trillion proton-proton collisions were simulated at different collision energies.

The antitriton and ${ }^{3} \overline{\mathrm{He}}$ yields from this simulation were validated by comparing them to available accelerator data. This is also the first MC simulation to predict ${ }^{4} \overline{\mathrm{He}}$ yields, which can be compared to data from future experiments. This model could be useful in describing the formation of light antinuclei in a variety of systems for a large range of energies using a single energy-dependent coalescence parametrization.

\section{Coalescence Formation of Light Antinuclei}

\subsection{Coalescence of two antinucleons}

The production of light antinuclei in hadronic interactions is not well understood. One successful model used to describe this process is the coalescence model [13]. In the simple coalescence model, the fusion of an antiproton and an antineutron into an antideuteron is based on the assumption that any antiproton-antineutron pair within a sphere of radius $p_{0}$ in momentum space will coalesce to produce an antinucleus.

However, this simple model does not take into account effects like energy conservation, spin alignment, and angular correlations. To take into account the hadronic physics (energy and momentum conservation, angular correlations, event topography, antiproton-antineutron production asymmetry, etc.), MC hadronic event generators are used. Typical hadronic generators [19-25] do not produce (anti)deuterons. Therefore, an event-by-event coalescence model afterburner was created. The afterburner applies the coalescence condition to $\overline{p n}$ pairs on a per-event basis. (e.g., Refs. [4, 13, 26]). For each event, the momentum difference of each antinucleon pair is calculated 

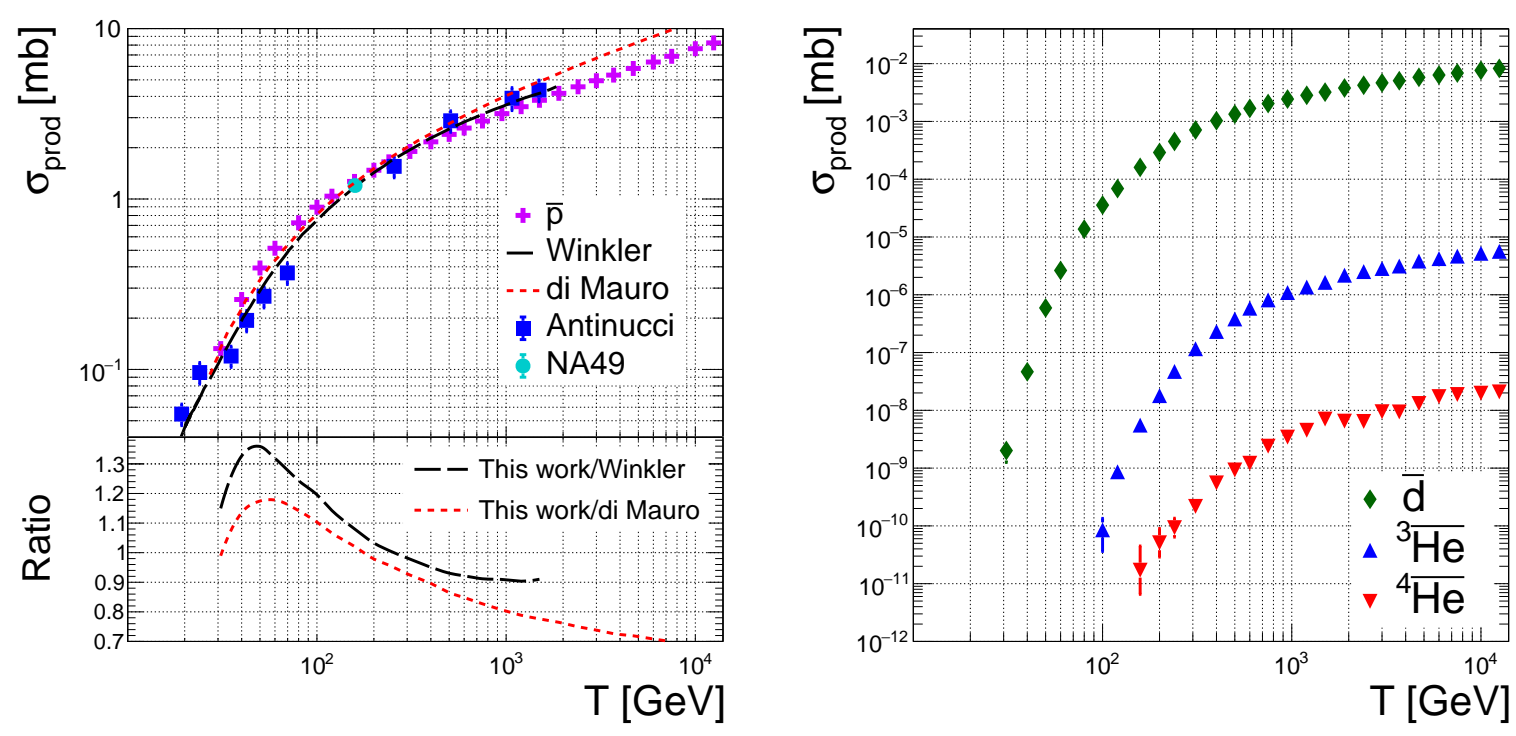

Figure 1: Production cross sections for (left) $\bar{p}$ and (right) light antinuclei in $p$ - $p$ collisions as function of collision kinetic energy $T[\mathrm{GeV}]$ (laboratory frame), using the coalescence mechanism at $120 \%$ of $p_{0, G}$. The $\bar{p}$ production cross sections are also compared to experimental data from Refs. [28, 29] and parametrizations from Winkler [30] and di Mauro [31, 32].

in their corresponding center-of-mass frame. If the momentum difference is smaller than the coalescence momentum $p_{0}$, a new particle is produced [2]. The $p_{0}$ is varied as a free parameter, and best-fit values are obtained by comparisons with the experimental data.

In Ref. [13], the formation of (anti)deuterons was studied using multiple MC event generators, and EPOS-LHC [19] was shown to be consistent with $\bar{p}$ production data in a wide range of energies. It was chosen as the event generator for this study as well. The $p_{0}$ parametrization for antideuteron production using EPOS-LHC was described by:

$$
p_{0}(T)=\frac{A}{1+\exp (B-\ln (T / C))}
$$

where $T$ is the collision kinetic energy in $\mathrm{GeV}$ and the parameters $A, B$, and $C$ were determined to be $89.6 \pm 3.0 \mathrm{MeV} / c, 6.6 \pm 0.88$, and $0.73 \pm 0.10$, respectively [13]

\subsection{Coalescence of larger antinuclei}

This work extends the event-by-event coalescence mechanism of formation of two-particle nuclei to estimate the production of larger antinuclei (up to ${ }^{4} \overline{\mathrm{He}}$ ). Two simple scenarios were taken into account [2]:

i) Simultaneous coalescence.-An $N$-particle antinucleus is formed by simultaneously coalescing $N$ antiparticles, where each antiproton and antineutron pair has to fulfill the aforementioned coalescence condition.

ii) Iterated coalescence.-Antiprotons or antineutrons are iteratively added to a multi-antinucleon state if they fulfill the two-particle coalescence condition. 

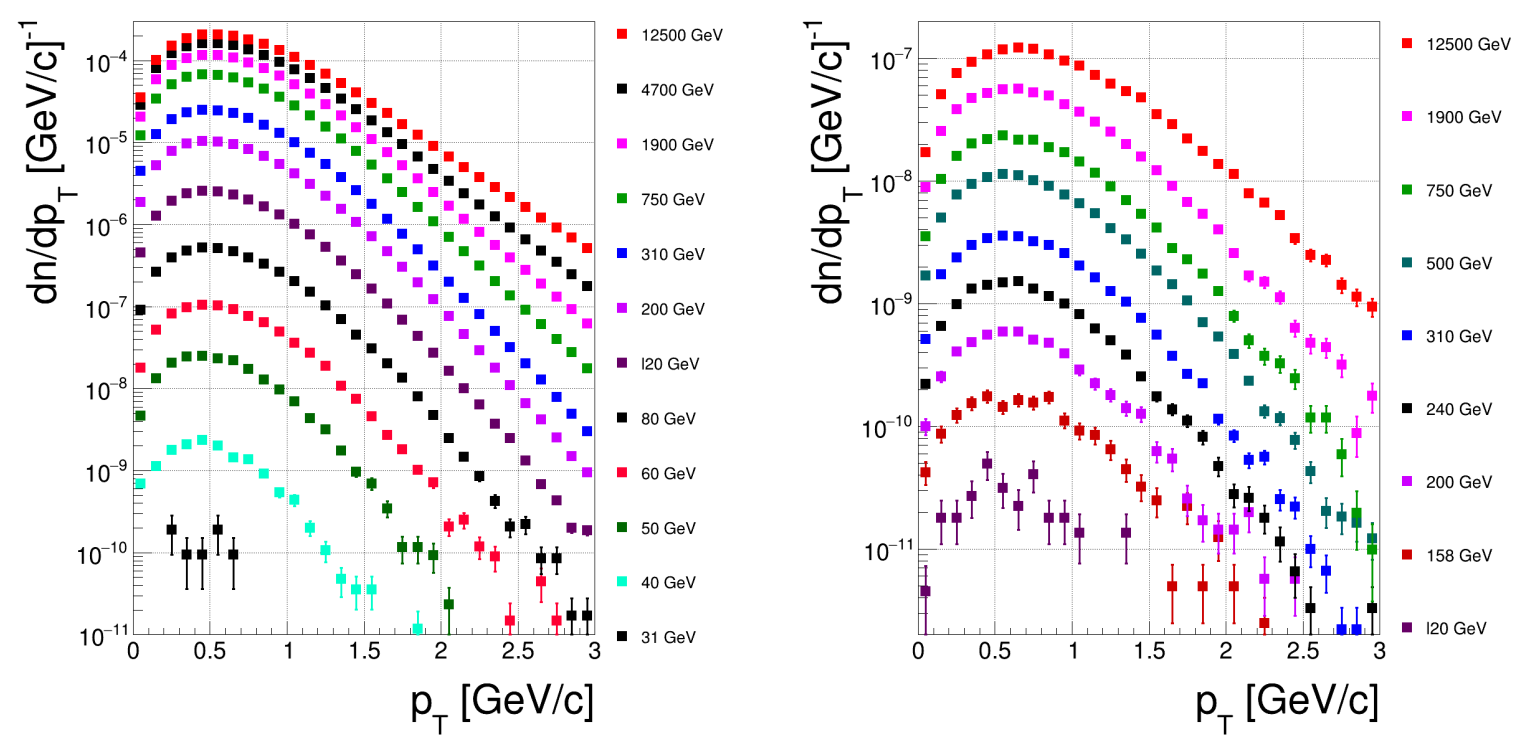

Figure 2: Antinuclei spectra as function of transverse momentum $p_{T}(\mathrm{GeV} / c)$ are plotted for selected CR energies (laboratory frame), using the coalescence mechanism at $120 \%$ of $p_{0, G}$ : (left) $\bar{d}$ spectra and (right) ${ }^{3} \overline{\mathrm{He}}$ spectra.

For a systematic study of the dependence of antinuclei production on $p_{0}$, seven different values of $p_{0}$ for each collision energy were used in this work. These seven values include the initial value of $p_{0}$ specific to that collision kinetic energy, from the $\bar{d}$ parametrization developed by Gomez et $a l$. For the rest of this study, this initial value is referred to as $p_{0, G}$. The remaining six values are $70 \%, 80 \%, 90 \%, 110 \%, 120 \%$, and $130 \%$ of $p_{0, G}$.

Proton-proton interactions were simulated using CRMC [27] at 27 logarithmically-spaced collision energies between $31 \mathrm{GeV}$ and $12.5 \mathrm{TeV}$ in the laboratory frame. Figure 1 (right) shows the total production cross section of different antiparticles as a function of collision energy (in the c.m. frame). The production of antiprotons increases with energy, and eventually saturates at high energy (approximately $1 \mathrm{TeV}$ ). As expected, a clear trend is observed that as the number of antinucleons in the final state increases, the production cross section of the final state particle decreases by almost an order of magnitude.

Figure 1 (left) shows a large gap in the $\bar{p}$ production cross section data in the few-hundred GeV range near the production thresholds. More experimental data in this region are crucial, as this is also the dominant region for the production of antinuclei in cosmic-ray interactions. Latest results from the NA61/SHINE experiment at CERN-SPS at $p_{\text {lab }}=158 \mathrm{GeV} / c$ [35] and analysis of new large datasets are very important. Figure 2 shows the predicted production yields of $\bar{d}$ and ${ }^{3} \overline{\mathrm{He}}$ as a function of transverse momentum $p_{T}$, for selected collision energies in the laboratory frame.

Since $p$ - $p$ collisions contribute $60 \%-70 \%$ of the total antinuclei source terms $[3,11]$, only those were simulated for this study. The remaining contributions ( $p-\mathrm{He}, \mathrm{He}-p$, and $\mathrm{He}-\mathrm{He}$ ) were estimated by scaling the parametrization developed in Ref. [32]. 

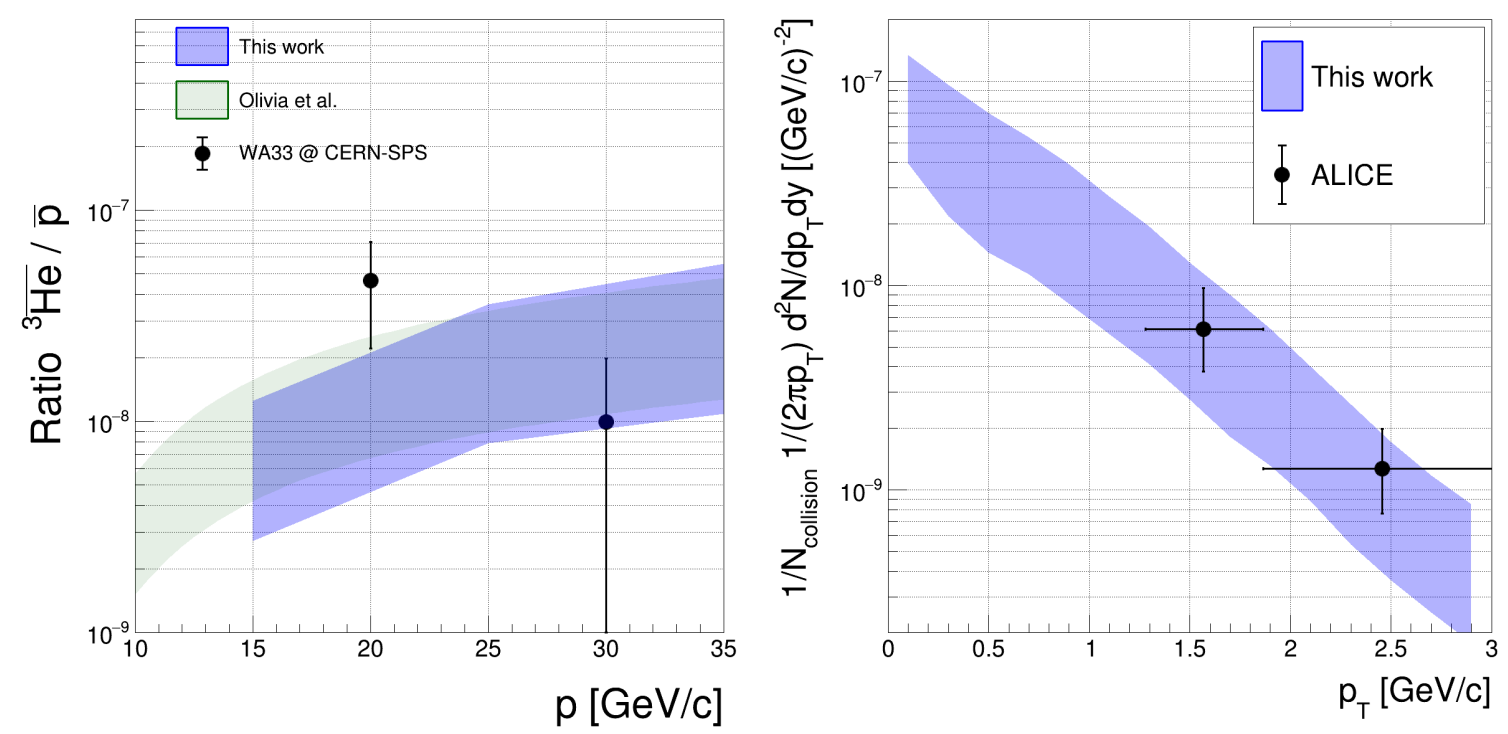

Figure 3: (Left) The invariant production cross section ratio ${ }^{3} \overline{\mathrm{He}} / \bar{p}$ as function of momentum $p[\mathrm{GeV} / c]$ in the laboratory frame for $p-\mathrm{Be}$ at $p_{\mathrm{lab}}=200 \mathrm{GeV} / c$. The uncertainty band was estimated by varying the coalescence parameter from $p_{0, G}(59 \mathrm{MeV} / c)$ to $130 \%$ of $p_{0, G}(77 \mathrm{MeV} / c)$. (Right) Number density of ${ }^{3} \overline{\mathrm{He}}$ production from coalescence mechanism for $p$ - $p$ interactions at $\sqrt{s}=7 \mathrm{TeV}$, along with ALICE results from Ref. [40]. The uncertainty band was estimated by varying the coalescence parameter from $p_{0, G}(90 \mathrm{MeV} / \mathrm{c})$ to $130 \%$ of $p_{0, G}(116 \mathrm{MeV} / c)$.

\section{Validating the multi-particle coalescence approach}

\subsection{Comparison with $\bar{p}$ production data}

Since the predicted antinuclei fluxes from cosmic-ray propagation models are highly correlated with antiproton production in proton-proton interactions, it is important to correctly simulate the antiproton production. The $\bar{p}$ total production cross sections from this study were compared with data at different collision energies [28, 29] in Figure 1 (left), and found to be within the uncertainties. The $\bar{p}$ differential production cross sections as function of kinetic energy were then compared to the latest parametrizations at collision energies of 20 and $450 \mathrm{GeV} / c$ in Ref. [2].

\subsection{Comparison with $\bar{d}, \bar{t}$ and ${ }^{3} \overline{\mathrm{He}}$ production data}

Because of a lack of light-antinuclei production data for $p$ - $p$ collisions at low energies near the production threshold, a direct comparison was not possible. However, comparison with $p$-A collisions (where A is a light antinucleus) can produce a target-independent parametrization for the production of light antinuclei, i.e., the model could be used to describe any $p$-A collisions. $\bar{t} / \bar{p}$ and ${ }^{3} \overline{\mathrm{He}} / \bar{p}$ ratios have been measured in $p$ - $\mathrm{Al}$ and $p$-Be collisions at beam momentum of $200 \mathrm{GeV} / c[37-$ 39]. The predictions of this model are compared to $p$-Be data in Figure 3 (left), and to $p$-Al data in Ref. [2]. The uncertainty bands were estimated by varying the coalescence parameter from $p_{0, G}$ $(59 \mathrm{MeV} / c)$ to $130 \%$ of $p_{0, G}(77 \mathrm{MeV} / c)$. In magnitude and shape, it nearly overlaps with the uncertainty band from the analytical model [39] and is in agreement with the data. 
$p$ - $p$ collisions were simulated at $\sqrt{s}=7 \mathrm{TeV}$ to compare with the latest ${ }^{3} \overline{\mathrm{He}}$ ALICE data [40]. The $p_{0}$ was again varied from $p_{0, G}(90 \mathrm{MeV} / c)$ to $130 \%$ of $p_{0, G}(116 \mathrm{MeV} / c)$ to simulate an uncertainty band. The ${ }^{3} \overline{\mathrm{He}}$ (shown in Figure 3 right) and $\bar{t}$ production (shown in Ref. [2]) are within $10 \%-30 \%$ of the yield predicted by using $p_{0, G}$. The model was also compared to the $\bar{d}$ production data at $\sqrt{s}=13 \mathrm{TeV}$ [41]. The uncertainty band for the model was again estimated by varying the coalescence parameter from $p_{0, G}(90 \mathrm{MeV} / c)$ to $130 \%$ of $p_{0, G}(116 \mathrm{MeV} / c)$. The $\bar{d}$ production data was found to be within $10 \%-20 \%$ of the yield predicted by using $p_{0, G}$ [2].

However, using $p_{0, G}$ as the coalescence parameter underpredicted the ${ }^{3} \overline{\mathrm{He}}$ production cross sections by $10 \%-20 \%$ at both high-energy and low-energy interactions. [2] The subsequent $\bar{d},{ }^{3} \overline{\mathrm{He}}$ and ${ }^{4} \overline{\mathrm{He}}$ cross sections, and the cosmic-ray flux discussion use an uncertainty band with the lower edge corresponding to $p_{0, G}$ and the upper edge corresponding to $130 \%$ of $p_{0, G}$ [2].

\section{Propagation of Antinuclei in the Galaxy}

The $\bar{d},{ }^{3} \overline{\mathrm{He}}$ and ${ }^{4} \overline{\mathrm{He}}$ differential production cross sections were used to calculate the local source terms $\mathrm{Q}_{\mathrm{sec}} \mathrm{using}[6,44,45]$ :

$$
\mathrm{Q}_{\mathrm{sec}}\left(E_{\mathrm{A}}\right)=4 \pi n_{\mathrm{H}} \int_{E_{t h}}^{\infty} \mathrm{d} E \phi_{p}(E) \frac{\mathrm{d} \sigma_{\mathrm{A}}}{\mathrm{d} E_{\mathrm{A}}}\left(E, E_{\mathrm{A}}\right)
$$

where $n_{H}$ is the number density of hydrogen nuclei in the ISM which was set to 0.9 atoms $/ \mathrm{cm}^{3}$. Data from from AMS-02 is used to obtain the flux of cosmic-ray protons $\phi_{p}(\mathrm{E})$ at the selected energies [5]. The secondary antiparticle source terms are presented in Figure 4 (left). Both $\bar{d}$ and ${ }^{3} \overline{\mathrm{He}}$ source terms are lower than the predictions by Poulin et al. [6] by an order of magnitude in the low kinetic energy region (less than $10 \mathrm{GeV}$ ). Because of low statistics, the ${ }^{4} \overline{\mathrm{He}}$ source term is shown only from $4-20 \mathrm{GeV}$, where it is in agreement with Poulin et al. As ${ }^{4} \overline{\mathrm{He}}$ production is extremely rare in $p-p$ collisions, being able to predict the ${ }^{4} \overline{\mathrm{He}}$ source term using MC simulations was only possible with a massive amount of computing power.

To model the propagation of antinuclei, software developed by Poulin et al. [6, 47, 48] was used with the MED propagation model [46]. The predicted antinuclei fluxes are shown in Figure 4 (right). The uncertainty bands shown for the fluxes from this work are due to the uncertainty in the coalescence parameter.

The predicted $\bar{p}$ flux exceeds the AMS-02 data by $20 \%-30 \%$ in the low kinetic energy region $(1-5 \mathrm{GeV})$. This can be explained by comparing the EPOS-LHC $\bar{p}$ production cross sections used in this work with the di Mauro parametrization used by Poulin et al. (Figure 1 left). The ratio (EPOS-LHC to di Mauro) goes up to 1.2 at low energies. Since low-energy collisions are the main source of antinuclei in cosmic-ray interactions, the overproduction of $\bar{p}$ in EPOS-LHC is the reason behind the excess $\bar{p}$ flux in this study. It is important to note that the $p_{0}$ parametrization of Gomez et al. [13] already absorbs this difference in $\bar{p}$ production between EPOS-LHC and experimental data. Hence, the excess $\bar{p}$ flux does not affect this study's predicted fluxes of the heavier antinuclei.

The predicted secondary $\bar{d}$ flux is very close to the flux from [6]. The predicted secondary ${ }^{3} \overline{\mathrm{He}}$ flux is consistently lower than the fluxes from both [6] and [3] by almost an order of magnitude. The ${ }^{4} \overline{\mathrm{He}}$ source term as well as the predicted secondary flux is shown only from $4-20 \mathrm{GeV}$, and they both agree with [6] within the uncertainties. 

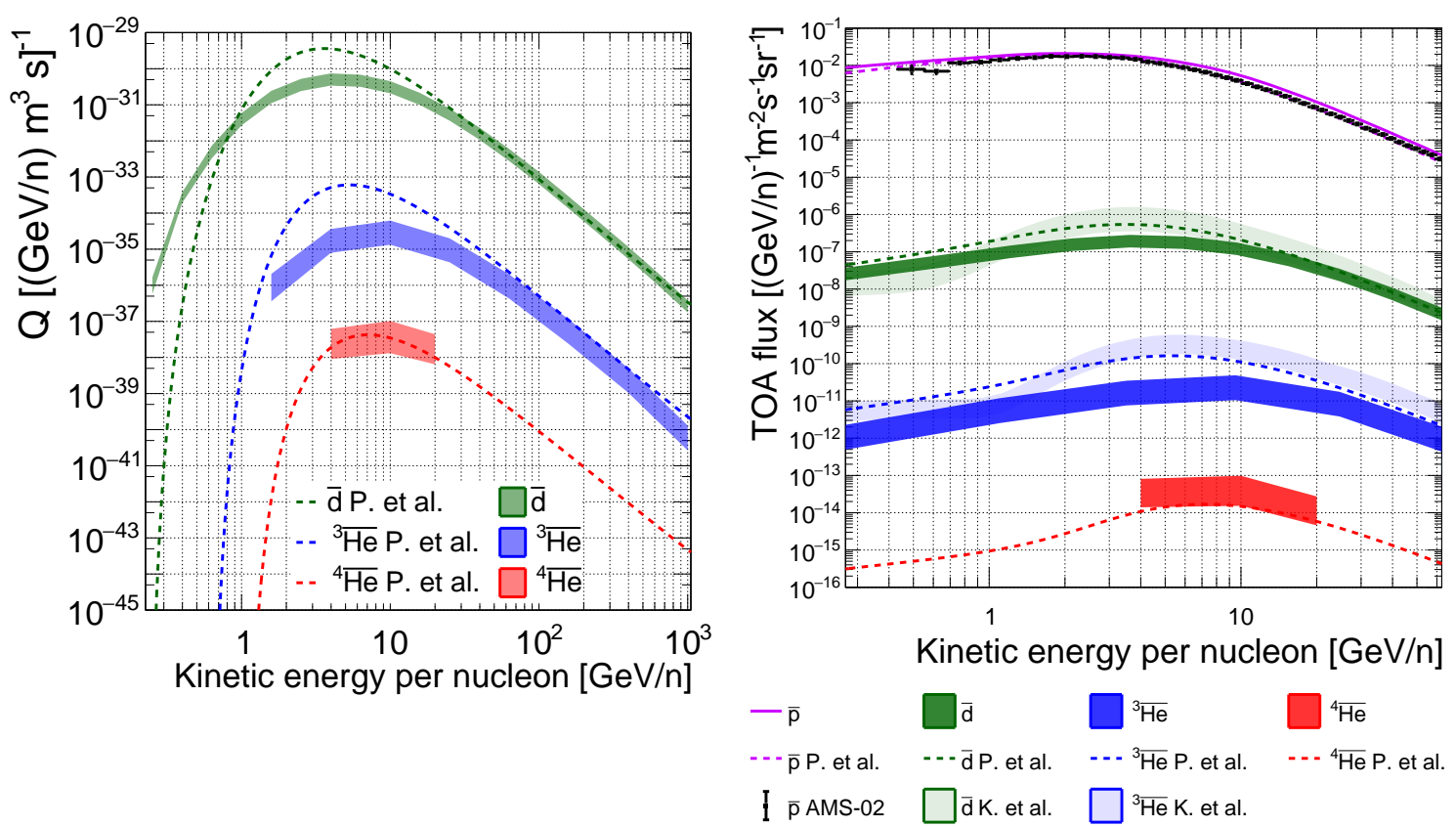

Figure 4: Left: local source terms, and Right: the predicted top-of-atmosphere fluxes, propagated using the MED propagation model [46], for the secondary production of $\bar{d},{ }^{3} \overline{\mathrm{He}},{ }^{4} \mathrm{He}$. These are compared to Poulin et al. [6] and Korsmeier et al. [3]. The uncertainty bands in both plots for this work represent the uncertainty in the coalescence parameter. The $\bar{p}$ flux is also compared to AMS-02 data [5].

The differences in the antinuclei fluxes between this study and Ref. [6] can be traced to the differences in the source terms in Figure 4 (left). The smaller predicted source terms by this study at lower energies is a consequence of the energy-dependent $p_{0}$.

\section{Conclusions}

A multiparticle coalescence model was developed to simulate the interaction of cosmic rays with the ISM, to produce light antinuclei in $p$-A collision simulations. The antinuclei production cross sections were successfully validated with available experimental data. For the first time, it was possible to simulate the production of ${ }^{4} \overline{\mathrm{He}}$ using MC. The lack of high-precision proton-proton data at lower energies remains a crucial gap and affects the CR background predictions.

The antinuclei produced by the model were propagated in the Galaxy to predict the top-ofthe-atmosphere secondary fluxes, which were compared to previous studies which use numerical scaling techniques to estimate the antinuclei production. The coalescence method predicts about an order-of-magnitude lower antideuteron and antihelium fluxes than the numerical scaling models.

\section{Acknowledgments}

A. S., A. D., D. G., and P. v. D. would like to thank the National Science Foundation (Grant No. 1551980). C. K. thanks the DAAD RISE fellowship. They also thank the Open Science Grid and the University of Hawaii's Mana HPC for access to high-throughput computing resources. 


\section{References}

[1] P. von Doetinchem et al., JCAP 08 (2020) 035.

[2] A. Shukla et al., Phys. Rev. D 102, 063004, 2020.

[3] M. Korsmeier et al., Phys. Rev. D, 97(10):103011, 2018.

[4] A. Ibarra et al., Phys. Rev. D, 88:023014, 2013.

[5] M. Aguilar et al. Phys. Rev. Lett., 117:091103, Aug 2016.

[6] V. Poulin et al., Phys. Rev. D, 99:023016, Jan 2019.

[7] R. P. Duperray et al., Phys. Rev. D, 68:094017, Nov 2003.

[8] S. T. Butler and C. A. Pearson. Phys. Rev., 129:836-842, Jan 1963.

[9] L. Csernai and J. I. Kapusta. Physics Reports, 131(4):223 - 318, 1986.

[10] A. J. Baltz et al., Phys. Lett. B, 325(1):7 - 12, 1994.

[11] D. Gomez. Ph.D. thesis Deuteron and antideuteron production in galactic cosmic-rays. 26 Apr 2019.

[12] Y. C. Ding et al., JCAP, 2019 (06):004-004, Jun 2019.

[13] D. Gomez-Coral et al., Phys. Rev. D, 98(2):023012, 2018.

[14] A. Schwarzschild and Č. Zupančič. Phys. Rev., 129:854-862, Jan 1963.

[15] P. Chardonnet et al., Phys. Lett. B, 409(1-4):313-320, Sep 1997.

[16] A. Ibarra et al., Phys. Rev. D 88, 023014

[17] A. Ibarra et al., JCAP 1302 (2013) 021.

[18] M. Kadastik et al., Phys. Lett. B 683:248-254, 2010.

[19] T. Pierog et al., Phys. Rev. C, 92(3):034906, 2015.

[20] S. Ostapchenko. Nucl. Phys. B - Proceedings Supplements, 151:147-150, 2006.

[21] T. Sjostrand et al., Computer Physics Communications, 178:852-867, 2008.

[22] S. Agostinelli et al. Nuclear Instruments and Methods in Physics Research A, 506(3):250-303, 2003.

[23] J. Allison et al., IEEE Transactions on Nuclear Science, 53:270-278, 2006.

[24] A. Galoyan and V. Uzhinsky. Hyperfine Interaction, 215(1-3):69-76, 2013.

[25] E. J. Ahn et al., Phys. Rev. D, 80:094003, 2009.

[26] M. Kadastik et al., Phys. Lett. B, 683:248-254, 2010.

[27] T. Pierog et al., Cosmic Ray Monte Carlo package.

[28] T. Anticic et al., Eur. Phys. J. C, 65(1-2), Nov 2009.

[29] M. Antinucci et al., Lett. Nuovo Cim. 6: No. 4, 121-128(27 Jan 1973)., 11973.

[30] M. W. Winkler. JCAP, 1702(02):048, 2017.

[31] M. di Mauro et al., Phys. Rev. D, 90:085017, Oct 2014.

[32] A. Reinert and M. W. Winkler. JCAP, 1801(01):055, 2018.

[33] S. Acharya et al. (ALICE Collaboration). Phys. Rev. C, 97(2):024615, 2018

[34] V. V. Abramov et al., Soviet Journal of Nuclear Physics, 45:5:845-851, May 1987.

[35] A. Aduszkiewicz et al., Eur. Phys. J. C, 77(10), Oct 2017.

[36] M. Korsmeier et al., Phys. Rev. D, 97(10):103019, 2018.

[37] W. Bozzoli et al., Nucl. Phys. B, 144(2):317 - 328, 1978.

[38] W. F. Baker et al., Phys. Lett. B, 51(3):303 - 305, 1974.

[39] J. Feng et al., 35th International Cosmic Ray Conference, 2017.

[40] S. Acharya et al., (ALICE Collaboration). Phys. Rev. C, 97:024615, Feb 2018.

[41] S. Acharya et al., (ALICE Collaboration). Eur. Phys. J. C 80 (2020) 889, 2020.

[42] S. Acharya et al., (ALICE Collaboration). Nucl. Phys. A, 971:1-20, Mar 2018.

[43] S. Acharya et al., (ALICE Collaboration). Phys. Rev. C, 101(4), Apr 2020.

[44] K. Blum et al., Phys. Rev. D, 96:103021, 2017.

[45] V. Duperray et al., European Physical Journal A Volume 16, pages 27-34, 2003.

[46] F. Donato et al., Phys. Rev. D, 69(6), Mar 2004.

[47] M. Boudaud et al., JCAP, 2015(05):013-013, May 2015.

[48] G. Giesen et al., JCAP, 2015(09):023-023, Sep 2015.

[49] R. Pordes et al., The Open Science Grid. 78:012057, 2007.

[50] I. Sfiligoi et al., The pilot way to grid resources using glideinwms. 2:428-432, 2009. 